\title{
CORRECTION
}

\section{Correction to: Role of total lung stress on the progression of early COVID-19 pneumonia}

Silvia Coppola , Davide Chiumello ${ }^{1}$, Mattia Busana², Emanuele Giola', Paola Palermo², Tommaso Pozzi ${ }^{1}$, Irene Steinberg ${ }^{2}$, Stefano Roli ${ }^{1}$, Federica Romitti ${ }^{2}$, Stefano Lazzari ${ }^{2}$, Simone Gattarello², Michela Palumbo ${ }^{2}$, Peter Herrmann², Leif Saager ${ }^{2}$, Michael Quintel ${ }^{2,3}$, Konrad Meissner $^{2}$, Luigi Camporota ${ }^{4}$, John J. Marini ${ }^{5}$, Stefano Centanni ${ }^{6}$ and Luciano Gattinoni $2^{*}$ (1)

C 2021 Springer-Verlag GmbH Germany, part of Springer Nature

\section{Correction to: Intensive Care Med (2021) 47:1130-1139} https://doi.org/10.1007/s00134-021-06519-7

The original version of this article unfortunately contained mistakes in Table 2 (wrong confidence interval data of "PEEP" and groups' size). Please find the corrected table below.
The authors apologize for the mistake.

*Correspondence: gattinoniluciano@gmail.com

2 Department of Anesthesiology, Medical University of Göttingen,

University Medical Center Göttingen, Robert Koch Straße 40,

37075 Göttingen, Germany

Full author information is available at the end of the article

\section{Springer}


Table 2 Gas-exchange and PEEP test in the ARDS subgroups

\begin{tabular}{|c|c|c|c|c|c|c|c|}
\hline & Overall $(n=140)$ & No ARDS $(n=17)$ & $\begin{array}{l}\text { Mild ARDS } \\
(n=45)\end{array}$ & $\begin{array}{l}\text { Mild-moderate } \\
\text { ARDS }(n=33)\end{array}$ & $\begin{array}{l}\text { Moderate- } \\
\text { severe ARDS } \\
(n=36)\end{array}$ & $\begin{array}{l}\text { Severe ARDS } \\
(n=9)\end{array}$ & $p$ value \\
\hline \multicolumn{8}{|l|}{ Gas exchange } \\
\hline $\mathrm{PaO}_{2}(\mathrm{mmHg})$ & 104 [79-140] & 226 [187-259] & 143 [102-151] & 112 [100-123] & 83.2 [75.9-93.6] & 65.1 [59.3-69] & $<0.001^{*}$ \\
\hline $\mathrm{FiO}_{2}$ & $0.6[0.6-0.7]$ & $0.6[0.6-0.7]$ & $0.6[0.5-0.7]$ & $0.7[0.5-0.7]$ & $0.7[0.6-0.7]$ & $0.8[0.7-0.8]$ & $<0.001^{*}$ \\
\hline $\mathrm{PaCO}_{2}(\mathrm{mmHg})$ & 37 [33.9-40.7] & $38.3[32.6-42.0]$ & 35.9 [32.6-39] & $38.8[35-41]$ & 36.5 [34.8-40.8] & 39 [34.2-39.9] & 0.37 \\
\hline $\mathrm{pH}$ & 7.44 [7.42-7.47] & 7.44 [7.42-7.46] & $7.45[7.43-7.48]$ & 7.44 [7.43-7.47] & $7.44[7.42-7.46]$ & 7.43 [7.42-7.45] & 0.52 \\
\hline $\begin{array}{l}\text { Respiratory rate } \\
\text { (bpm) }\end{array}$ & 20 [18-24] & 16 [15-20] & 21 [18-23] & 21 [18-24] & 21 [18-25] & 20 [18-20] & $0.039^{*}$ \\
\hline PEEP $\left(\mathrm{cmH}_{2} \mathrm{O}\right)$ & $7.5[7.5-10]$ & $10[7.5-10]$ & $8[7.5-10]$ & $8[7.5-10]$ & $9[7.5-10]$ & 10 [10-10] & 0.24 \\
\hline $\begin{array}{l}\text { Esophageal } \\
\text { pressure swing } \\
\left(\mathrm{cmH}_{2} \mathrm{O}\right)\end{array}$ & $7[5-10]$ & $6[5-7]$ & $7[5-9]$ & $7[4-9]$ & $9[7-12]$ & $7[5-7]$ & 0.06 \\
\hline $\begin{array}{c}\text { Total stress } \\
\left(\mathrm{cmH}_{2} \mathrm{O}\right)\end{array}$ & 14 [11.5-16.3] & $13[11.8-15]$ & 13.2 [11.3-16] & $13.8[11-16]$ & 15.2 [12.9-18.6] & 14 [13.3-16.8] & 0.12 \\
\hline Borg dyspnea scale & $0[0-1]$ & $0[0-1]$ & $0[0-0]$ & $0[0-0]$ & $0[0-1]$ & $0[0-1]$ & 0.34 \\
\hline \multicolumn{8}{|l|}{ PEEP-test } \\
\hline $\begin{array}{l}\mathrm{SpO}_{2} \text { at } 0 \mathrm{CmH}_{2} \mathrm{O} \\
(\%)\end{array}$ & 97 [93-99] & 98 [94-100] & 98 [96-99] & 98 [95-99] & 95 [93-98] & 91 [88-94] & $0.003^{*}$ \\
\hline $\begin{array}{l}\mathrm{SpO}_{2} \text { at } 10 \mathrm{cmH}_{2} \mathrm{O} \\
(\%)\end{array}$ & 98 [96-99] & 99 [98-100] & 99 [97-100] & 98 [97-99] & 97 [96-98] & 94 [94-98] & $0.006^{*}$ \\
\hline $\begin{array}{l}\text { Respiratory rate at } \\
\mathrm{O} \mathrm{CmH}_{2} \mathrm{O}(\mathrm{bpm})\end{array}$ & 22 [18-28] & 18 [15-20] & 22 [18-28] & 23 [20-28] & 22 [19-27] & 24.5 [22-27] & $0.010^{*}$ \\
\hline $\begin{array}{l}\text { Respiratory rate at } \\
10 \mathrm{cmH}_{2} \mathrm{O}(\mathrm{bpm})\end{array}$ & 20 [16-24] & 16 [15-18] & 20 [17-22] & 22 [18-25] & 20 [17-28] & 19.5 [18-21] & $0.007^{*}$ \\
\hline $\begin{array}{l}\text { Esophageal pres- } \\
\text { sure swing at } 0 \\
\mathrm{cmH}_{2} \mathrm{O}\left(\mathrm{cmH}_{2} \mathrm{O}\right)\end{array}$ & 8 [6-12] & $7[5-7]$ & $8[5-10]$ & 8 [5-12] & 8 [7-15] & 8.8 [6-11] & 0.36 \\
\hline $\begin{array}{l}\text { Esophageal pres- } \\
\text { sure swing at } 10 \\
\mathrm{cmH}_{2} \mathrm{O}\left(\mathrm{cmH}_{2} \mathrm{O}\right)\end{array}$ & $7[5-10]$ & 7.5 [6-9] & $7[5-9]$ & 7 [4-9] & $9[7-12]$ & $7[6-9]$ & 0.07 \\
\hline $\begin{array}{l}\text { Total stress at } 0 \\
\mathrm{CmH}_{2} \mathrm{O}\left(\mathrm{cmH}_{2} \mathrm{O}\right)\end{array}$ & 8 [6-12] & $7[5-10]$ & $8[5-10]$ & 8 [5-12] & $8[7-15]$ & $9[6-11]$ & 0.36 \\
\hline $\begin{array}{l}\text { Total stress at } 10 \\
\qquad \mathrm{cmH}_{2} \mathrm{O}\left(\mathrm{cmH}_{2} \mathrm{O}\right)\end{array}$ & 14 [12-17] & 14.5 [13-16.3] & 14 [12-16] & 14 [11.3-16.3] & 16 [14-18.5] & 14 [13.4-15.6] & 0.07 \\
\hline
\end{tabular}

\section{Author details}

${ }^{1}$ Department of Anesthesiology and Intensive Care, ASST Santi e Paolo Hospital, University of Milan, Milan, Italy. ${ }^{2}$ Department of Anesthesiology, Medical University of Göttingen, University Medical Center Göttingen, Robert Koch Straße 40, 37075 Göttingen, Germany. ${ }^{3}$ Department of Anesthesiology, Intensive Care and Emergency Medicine Donau-Isar-Klinikum Deggendorf, Deggendorf, Germany. ${ }^{4}$ Department of Adult Critical Care, Guy's and St Thomas' NHS Foundation Trust, Health Centre for Human and Applied Physiological Sciences, London, UK. ${ }^{5}$ Department of Pulmonary and Critical Care Medicine, University of Minnesota and Regions Hospital, St. Paul, MN, USA.
${ }^{6}$ Respiratory Unit, San Paolo Hospital, Dipartimento Scienze della Salute, Università degli Studi di Milano, Milan, Italy.

\section{Publisher's Note}

Springer Nature remains neutral with regard to jurisdictional claims in published maps and institutional affiliations.

Published online: 14 December 2021 